\title{
Effectiveness of Active Rehabilitation Program on Sports Hernia: Randomized Control Trial
}

\author{
Walid Ahmed Abouelnaga, PhD, PT, Nancy Hassan Aboelnour, PhD, PT
}

Physical Therapy Department for Surgery, Faculty of Physical Therapy, Cairo University, Giza, Egypt

Objective To determine whether an active rehabilitation program that involves repetitive effortful muscle contractions, including core stability, balancing exercises, progressive resistance exercises, and running activities, after a sports hernia, is effective.

Methods Forty soccer players with sports hernias were randomly divided into two equal groups: group A (active rehabilitation program) and group B (conventional treatment). The methods of assessment included a visual analog scale (VAS) and hip internal and external range of motion assessments. Group A received conventional treatment (heat, massage, transcutaneous electrical nerve stimulation, and mobilization) plus an active rehabilitation program, while group B received only conventional treatment. Three treatment sessions were given each week for 2 months. Evaluations were performed pre- and post-treatment.

Results A decrease in VAS was seen in both groups at the end of treatment, $80.25 \%$ in group A and $41.93 \%$ in group B. The difference between the two groups was statistically significant $(\mathrm{p}=0.0001)$, whereas there were no statistical differences in internal and external rotation between the groups at the end of treatment ( $p>0.05)$. After treatment, an improvement in outcome measures of group A compared to group B $(p=0.01)$ was seen. Thirteen patients in group A and only three patients in group B returned to sports activities without groin pain.

Conclusion Active rehabilitation was effective for sports hernia management measured by a decrease in pain and the return to sports.

Keywords Sport hernia, Visual analog scale (VAS), Active rehabilitation

\section{INTRODUCTION}

Hip and groin pain is problematic for athletes, particularly in sports involving high amounts of running, changes in direction and kicking [1]. Gilmore reported that sports hernias were present in $59 \%$ of patients who par- ticipated in soccer, $10 \%$ of rugby, $4 \%$ of racquet games, $4 \%$ of athletics, $2 \%$ of cricket, $2 \%$ of hockey, and $19 \%$ of alternative sports players [2], while in the United States, sports hernias were observed in $46 \%$ of patients who participated in soccer, $17 \%$ of hockey, $13 \%$ of football, and $24 \%$ of different sports, including swimming, tennis, bas-

Received October 2, 2018; Accepted December 27, 2018

Corresponding author: Walid Ahmed Abouelnaga

Physical Therapy Department for Surgery, Faculty of Physical Therapy, Cairo University, 7th Ahmed Elzayat St., Bein Al Sarayat, Giza 12613, Egypt. Tel: +20-100-566-6892, Fax: +20-23-761-7692, E-mail: Walidabolnaga@yahoo.com

ORCID: Walid Ahmed Abouelnaga (http://orcid.org/0000-0002-3050-351X); Nancy Hassan Aboelnour (http://orcid.org/0000-0003-1643-0606).

(c) This is an open-access article distributed under the terms of the Creative Commons Attribution Non-Commercial License (http://creativecommons.org/ licenses/by-nc/4.0) which permits unrestricted noncommercial use, distribution, and reproduction in any medium, provided the original work is properly cited. Copyright ( 2019 by Korean Academy of Rehabilitation Medicine 
ketball, and golf [3].

A sports hernia is a painful condition, noted for soreness and sharp pain at the groin area near the pubic tubercle at the onset. The symptoms reflect injuries to the outer inguinal ring, which becomes dilated with posterior wall deficiency of the inguinal canal (fascia transversalis,) and tears in the conjoint tendon [4]. Different hypotheses explain the possible causes of pain in sports hernia, including posterior wall distension can contribute for torn of conjoint tendon. It was proposed that outer inguinal ring dilation caused by tearing of the external oblique aponeurosis, in addition to conjoint tendon laceration, leading to impairment and fragility between the inguinal ligament and the conjoint tendon [5]. Others have suggested that either the ilioinguinal or genitofemoral nerve entrapment could give rise to the pain [6,7]. Entrapment of the ilioinguinal nerve caused by a hernia of the external oblique aponeurosis is called hockey groin [8]. However, nerve entrapment or stretching is, evidently, not the only reason for sports hernia pain because it is indistinguishable from nerve stretching experienced by athletes with torn conjoint tendons.

The expression of a sports hernia includes many structural abnormalities along with a set of secondary causes. The most commonly hypothesized cause is that sports hernias occur secondary to intensive hip muscle contractions and excessive use of flexion, extension, abduction, and adduction moments across the hip joint with twisting or hyperextension of the trunk during sports, leading to exaggerated movement of the pelvis that creates a shearing force over the symphysis pubis $[9,10]$. The force is further exaggerated by the imbalance between the two opposing forces acting on the pubis, the upward and oblique dragging of the abdominal muscles from one side and the downward and lateral dragging of the adductors on the other side. This disequilibrium of forces can cause injuries to both the abdominal and hip adductors [11]. The traction of hip adductors against the nonmoving lower limb may generate shearing forces across the hemipelvis and has been considered an additional cause of sports hernias [12].

Therefore, the ideal functional stability of the groin can be lost by this disequilibrium between the abdominal and hip muscles. Thus, a vigorous off-season conditioning program that puts more emphasis on strengthening the lower limbs, while ignoring the abdominal muscles, may lead to a pelvic balance disturbance and shortening of the hip flexors and adductors, which increase the opportunity for sports hernia development [8].

The diagnosis of sports hernias should include at least three of these five clinical findings: (1) tenderness at the insertion of the conjoint tendon, (2) tenderness at the inner inguinal ring, (3) dilated superficial inguinal ring without clinical signs of a hernia but with painful points at the hip adductors attachment, (4) hip adductor longus muscle pain at its origin, and (5) widespread inguinal distress extending to the perineum and femoral interior surface and intersecting the midline [13].

Management of sports hernias usually follows different strategies which depend on combined treatments encompassing medications, surgical interventions, and physical therapy $[14,15]$. Non-surgical intervention is the first recommended treatment and includes rest (6 to 8 weeks), NSAIDs, ice packs, massage, and exercises [12]. Limitations in hip range of motion (ROM) have been reported in athletes suffering from groin pain [16] as with the chronicity of sports hernias, there is a decrease in hip ROM for external and internal rotation [17]. It has been hypothesized that the hip-joint capsular twisting performed during participation in Australian Rules Football may produce movement restrictions like those created at the glenohumeral joint by repetitive throwing [18]. Restricted joint mobility can be managed with joint mobilization of the proper part of the joint capsule, usually the posterior, inferior, and lateral parts [19], and stretching is also commonly used during rehabilitation to decrease joint stiffness and increase ROM [20]. Vigorous exercises or stretching used in an attempt to increase the hip ROM should be avoided to prevent excessive pain [21].

Physical rehabilitation programs for sports hernias should mainly include core stabilization, strengthening of the hip adductors and abdominal muscles, balancing, and postural training exercises [12,19,21]. Core strength exercises involving muscle strengthening and balancing exercises are always used to reinforce and strengthen abdominal and pelvic muscles [22] because these muscles play a significant role in improving muscle flexibility, lumber stability, and body balance [23]. Several studies have reported the importance of core stability and its effects to prevent sports injuries and improve athletic activity and performance through inhibition of false motion patterns and the maintenance of good postural align- 
ment [24].

Active physical training aiming to strengthen hip adductors and abdominal muscles and improve endurance, balance, and coordination between the hip and trunk muscles was found to be an effective method for sports hernia management [25]. In a randomized experimental study comparing active physical training and passive therapy in athletes with groin pain, active exercise training was found to be more effective and have greater benefits to return of athletes to their pre-injury activity levels faster [26].

Rehabilitation and exercise therapy for sports hernias usually depends on the past experience of the therapist rather than on an integrated protocol $[14,15]$. Therefore, this study was designed to evaluate the efficacy of active rehabilitation for the management of sports hernias.

\section{MATERIALS AND METHODS}

\section{Subjects}

Forty soccer players diagnosed with sports hernias participated in this study. The diagnosis was made by magnetic resonance imaging, which revealed disruption of the distal rectus abdominis/adductor aponeurosis, and was correlated with information provided from the patient's medical history and physical examination. The athletes complained of a dull and diffuse pain in the groin, radiating to the lower abdomen and proximal thigh. The pain was worsened by coughing, sneezing, or other Valsalva-type maneuvers. During the examination, the abdominal obliques, transversus abdominis, conjoint tendon/rectus abdominis, and adductor longus were palpated. The diagnosis of sports hernias should include at least three of these five clinical findings: (1) pinpoint tenderness over the pubic tubercle at the point of insertion of the conjoint tendon, (2) palpable tenderness over the deep inguinal ring, (3) pain and/or dilation of the external ring with no obvious hernia evident, (4) pain at the origin of the adductor longus tendon, and (5) dull, diffuse pain in the groin, often radiating to the perineum and inner thigh or across the midline. Two special tests were done to confirm the diagnosis, resisted sit-ups and single or bilateral resisted leg adduction tests. To be included in the study, the athletes had to be male, aged 18-25 years, have groin pain for at least 2 months due to playing sports, and exhibit at least three of the five clinical find- ings mentioned above. The exclusion criteria included a palpable inguinal or femoral hernia; evidence of lumbar radiculopathy; sacroiliac dysfunction; nerve entrapments of the ilioinguinal, genitofemoral, or lateral femoral cutaneous nerves; hip joint disorders, such as osteoarthritis, osteochondritis dissecans, and bursitis; and femoroacetabular impingement.

\section{Design}

The study was a single-blinded randomized controlled clinical trial and was approved by the ethical committee of the Faculty of Physical Therapy, Cairo University. All patients provided informed consent before participating in the study. After inclusion, the patients were randomly divided into two equal groups. The randomization process was applied using the envelope method. Cards on which either 'conventional treatment' or 'active rehabilitation program' was written on them were sealed in envelopes. The envelopes were given to a staff physical therapist who was blinded to this study and she/he picked one envelope. Depending on the card selected, the participants were allocated to their respective group. Appointments to begin the allocated treatment were arranged and treatment was started within one week of randomization. The examining physical therapist was not involved in the randomization process and remained unaware of the treatment allocation. Athletes were instructed not to reveal their treatment allocation to the physical therapist during their assessment.

\section{Treatment}

All patients were evaluated before and after the end of the treatment. The visual analog scale (VAS) was used for pain assessment and a goniometer was used to assess the ROM of the hip internal and external rotation. Pain was assessed with Valsalva's maneuver, forcibly exhaling against a closed airway, and with a resisted sit-up and the mean was determined. Hip internal and external rotations were measured in the supine position with the hip and knee at $90^{\circ}$ flexion.

After the end of the treatment, the outcome measures of successful treatment were evaluated. Successful treatment was defined as no pain at palpation of the adductor tendons and the adductor insertions at the pubic bone and no pain during active adduction against resistance, no groin pain in connection with or after athletic activity 
in the same sport and at the same level of competition as before the onset of the groin pain, and return to the same sport and at the same level without groin pain. If all three measures were reached, the result was labeled excellent, if two measures were reached, the result was good, if one measure was reached, the result was fair, and if no measures were reached, the result was poor.

In this study, both groups received conventional treatment as described in Table 1. Conventional treatment included heat, transverse friction massage, transcutaneous electrical nerve stimulation (TENS) and mobilization. Both groups were instructed to perform stretching exercises included in the treatment program for the adductor, hamstring, and hip-flexor muscles on the days between the treatment days.

In addition, participants in group A received an active rehabilitation program, which included strengthening exercises for the hip and abdominal muscles, core stabilization, and balancing exercises (Table 2). The patients were instructed to perform the exercises included in the treatment program on the days between the treatment days.

Patients in both groups were not allowed to receive any other treatments for groin pain during the study period. Patients in both groups were instructed to return to a running program at the sixth week of the study, which progressed from slow jogging to straight sprints and finally ended with cutting. The progression of exercise was performed in the absence of pain during exercise, the acquisition of functional control, and the ability to complete a functional exercise or a set number of repetitions of an exercise.

\section{Statistical analyses}

Descriptive statistics and t-tests were conducted for the comparison of subject characteristics between the groups. t-tests were conducted to compare the mean values of VAS and ROMs between both groups and paired t-tests were conducted to compare the pre- and posttreatment mean values of the measured variables in each group. Chi-square tests were conducted to compare outcome measures between the groups. The level of significance for all statistical tests was set at $p<0.05$. All statistical tests were performed using the Statistical Package for Social Sciences (SPSS) version 19 for Windows (IBM SPSS, Armonk, NY, USA).

Table 1. Conventional treatment program

\begin{tabular}{|c|c|}
\hline & Description \\
\hline Heat & Hot packs for 10 minutes on painful groin area. \\
\hline Transverse friction massage & Applied for 10 minutes to painful area of adductor-tendon insertion into pubic bone. \\
\hline TENS & Applied for 30 minutes to painful area. \\
\hline Mobilization/techniques & $\begin{array}{l}\text { A) Anterior ilium rotation mobilization: Patient in prone lying position. Therapist } \\
\text { stands contralateral of ilium to be mobilized. Bottom hand grasps anterior distal } \\
\text { thigh (knee flexed or extended), bringing hip into extension. Anterior superior } \\
\text { force is applied over posterior ilium to induce anterior rotation of the innominate. } \\
\text { B) Posterior ilium rotation mobilization: Patient on side, lying facing the therapist, } \\
\text { hip and knee } 60^{\circ}-90^{\circ} \text {. Bottom hand makes contact over ischial tuberosity, top } \\
\text { hand makes contact over ASIS. Two parallel forces are applied with both hands to } \\
\text { induce posterior rotation of the innominate. } \\
\text { C) Hip anterior glide mobilization: Patient in prone lying position. Stabilizing hand } \\
\text { grasps anterior distal femur positioning hip in neutral and knee flexed } 90^{\circ} \text {. Mobi- } \\
\text { lizing hand contacts posterior proximal femur applying anterior force. } \\
\text { D) Hip posterior glide mobilization: Patient in supine lying position. Therapist stands } \\
\text { on opposite side of involved hip, places cephalad hand underneath ischium or } \\
\text { can uses a wedge. Position the hip in } 90^{\circ} \text { flexion and } 10^{\circ} \text { adduction. Caudal hand } \\
\text { contacts patella, exerting posterior force through long axis of femur. }\end{array}$ \\
\hline Stretching exercises & $\begin{array}{l}\text { For adductor muscles, hamstring muscles, and hip flexors, the contract-relax tech- } \\
\text { nique was used. The stretching was repeated three times and the duration of each } \\
\text { stretch was } 30 \text { seconds. }\end{array}$ \\
\hline
\end{tabular}

TENS, transcutaneous electrical nerve stimulation. 
Table 2. Active rehabilitation program

\begin{tabular}{|c|c|c|}
\hline 1-2 weeks & 2-6 weeks & 6-8 weeks \\
\hline $\begin{array}{l}\text { 1. Static adduction against soccer } \\
\text { ball placed between feet while } \\
\text { lying supine. Each adduction } 30 \\
\text { seconds, } 10 \text { repetitions. } \\
\text { 2. Static adduction against soccer } \\
\text { ball placed between knees while } \\
\text { lying supine. Each adduction } 30 \\
\text { seconds, } 10 \text { repetitions. } \\
\text { 3. Posterior pelvic tilting. } \\
\text { 4. Bridging on the floor. Five series } \\
\text { of } 10 \text { repetitions. } \\
\text { 5. Sitting on ball, positioning knee } \\
\text { and hips at } 90^{\circ} \text { with hands on } \\
\text { thighs while trying to maintain } \\
\text { pelvic and trunk stability. } \\
\text { 6. Abdominal sit-ups, both in st- } \\
\text { raightforward direction and in } \\
\text { oblique direction. Five series of } \\
10 \text { repetitions. } \\
\text { 7. Combined abdominal sit-up and } \\
\text { hip flexion, starting from supine } \\
\text { position and with soccer ball } \\
\text { placed between knees (folding } \\
\text { knife exercise). Five series of } 10 \\
\text { repetitions. } \\
\text { 8. Balance training on wobble board } \\
\text { for } 5 \text { minutes. }\end{array}$ & $\begin{array}{l}\text { 1. Cardiovascular warm up: bike or } \\
\text { elliptical. } \\
\text { 2. Leg abduction and adduction ex- } \\
\text { ercises lying on side. Five series } \\
\text { of } 10 \text { repetitions of each exercise. } \\
\text { 3. One-leg weight-pulling abduc- } \\
\text { tion/adduction standing. Five se- } \\
\text { ries of } 10 \text { repetitions for each leg. } \\
\text { 4. Abdominal sit-ups, both in st- } \\
\text { raightforward direction and in } \\
\text { oblique direction. Five series of } \\
\text { 10 repetitions. } \\
\text { 5. Bridging on ball: place a physio- } \\
\text { ball under legs and apply down- } \\
\text { ward pressure to the ball as the } \\
\text { legs straighten allowing the pel- } \\
\text { vis to rise from the surface. } \\
\text { 6. Hip conditioning and core sta- } \\
\text { bilization exercises: sitting on } \\
\text { the ball with the opposite upper } \\
\text { extremity placing opposing pres- } \\
\text { sure on raised knee while the } \\
\text { other upper extremity is raised in } \\
\text { the air for additional stabilizing } \\
\text { challenge. } \\
\text { 7. Quadriped hip extension with } \\
\text { neutral spine. Two series of } 15 \\
\text { repetitions. } \\
\text { 8. Quadriped alternating opposite } \\
\text { arm and leg extension with neu- } \\
\text { tral spine. Two series of } 15 \text { repeti- } \\
\text { tions. } \\
\text { 9. Half-kneeling with perturbations. } \\
\text { Three sets of } 30-60 \text { seconds for } \\
\text { each limb. } \\
\text { 10. Forward/backward walking lung- } \\
\text { es with medicine ball lift. Two } \\
\text { to three sets of } 10-15 \text { lunges for- } \\
\text { ward and } 10-15 \text { backward. } \\
\text { flexed. }\end{array}$ & $\begin{array}{l}\text { 1. Cardiovascular warm up on bike } \\
\text { or elliptical with higher speed } \\
\text { and resistance. } \\
\text { 2. Clam exercise: the patient in side } \\
\text { lying position with the target hip } \\
\text { on top in } 30^{\circ} \text { flexion, externally } \\
\text { rotated and abducted. A resis- } \\
\text { tance band is used to perform } \\
\text { isometric contraction. Five series } \\
\text { of } 10 \text { repetitions. } \\
\text { 3. Standing adduction with leg } \\
\text { pulley. Attach cable to ankle, } \\
\text { perform adduction movement } \\
\text { standing next to machine. Five } \\
\text { series for } 10 \text { repetitions. } \\
\text { 4. Bridging coupled with lower ex- } \\
\text { tremity lift: the patient is on ball, } \\
\text { lifts one leg into the air while } \\
\text { keeping knee extended and trunk } \\
\text { stabilized. } \\
\text { 5. Front plank: align shoulders } \\
\text { with elbows and lift into forearm } \\
\text { plank keeping pelvis in align- } \\
\text { ment, then progress to placing } \\
\text { hands aligned with shoulders } \\
\text { and fingers pressing into surface } \\
\text { keeping pelvis aligned with plank } \\
\text { position. } \\
\text { 6. Side plank: lying on side, align } \\
\text { shoulder, elbow, hips and ankles } \\
\text { and raise up into plank position, } \\
\text { maintaining alignment. } \\
\text { 7. Pelvic stability on unstable sur- } \\
\text { face: the patient sits on an air } \\
\text { filled balance disc, maintains } \\
\text { balance while lifting one knee to- } \\
\text { ward chest, then lifts both knees. } \\
\text { Same exercise repeated with a } \\
\text { ball toss. } \\
\text { es with medicine ball lift. Two to } \\
\text { three sets of } 10-15 \text { lunges forward } \\
\text { and } 10-15 \text { backward. } \\
\text { 9. Single leg balance on } 360^{\circ} \text { bal- } \\
\text { flexed with ball toss. }\end{array}$ \\
\hline
\end{tabular}

\section{RESULTS}

A diagram of the study randomization is shown in Fig. 1.
A total of 55 male patients were admitted and assessed for eligibility. Fifteen of them were excluded because they did not meet the eligibility criteria, while 40 patients 


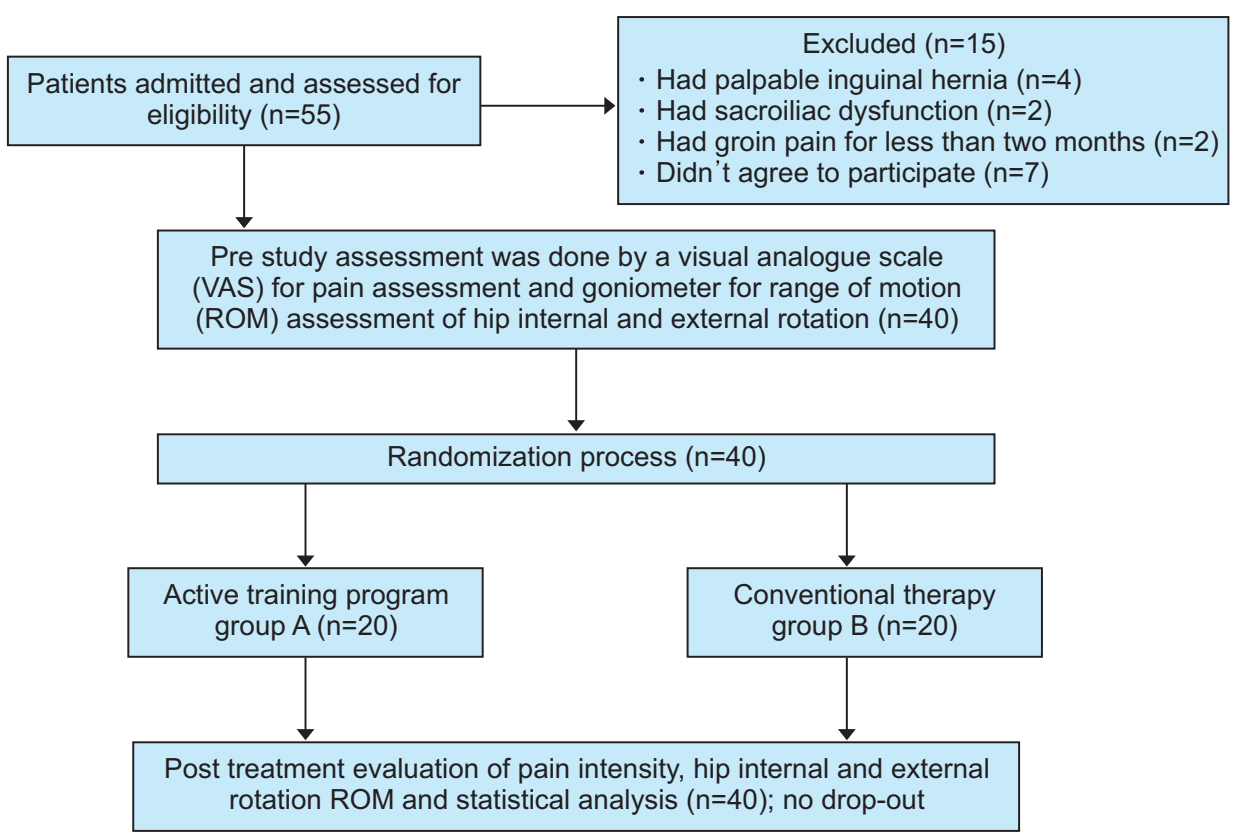

Fig. 1. Participant flowchart.

Table 3. Comparison of VAS, internal rotation, and external rotation ROM between groups A and B pre- and post-treatment

\begin{tabular}{lrcccc}
\hline & Group A & Group B & Mean diff. & t & p-value \\
\hline Pre-treatment & & & & & \\
$\quad$ VAS & $7.85 \pm 0.74$ & $7.75 \pm 0.71$ & 0.1 & 0.43 & 0.66 \\
\hline Internal rotation $\left(^{\circ}\right)$ & $32.00 \pm 1.58$ & $31.55 \pm 1.50$ & 0.45 & 0.92 & 0.36 \\
\hline External rotation $\left(^{\circ}\right)$ & $37.80 \pm 1.50$ & $37.85 \pm 1.46$ & -0.05 & -0.1 & 0.91 \\
\hline Post-treatment & & & & & \\
\hline VAS & $1.55 \pm 0.68$ & $4.50 \pm 0.60$ & -2.95 & -14.39 & $0.0001^{*}$ \\
\hline Internal rotation $\left(^{\circ}\right)$ & $39.10 \pm 0.71$ & $38.95 \pm 0.82$ & 0.15 & 0.61 & 0.54 \\
\hline External rotation $\left(^{\circ}\right)$ & $44.35 \pm 0.67$ & $44.00 \pm 1.21$ & 0.35 & 1.12 & 0.26 \\
\hline
\end{tabular}

Values are presented as mean \pm standard deviation.

VAS, visual analog scale; ROM, range of motion.

${ }^{*} \mathrm{p}<0.05$.

were found to be eligible to participate in the study. All patients completed the treatment program without withdrawal.

\section{Demographics}

The mean \pm standard deviation for age, weight, and height of group A were 26.2 $\pm 2.94,71.05 \pm 2.7$, and 169.9 \pm 3.3 and the values for group B were $26.75 \pm 3.02,70.45 \pm 3.21$, and $170.15 \pm 3.77$, respectively. There was no statistical difference between the groups $(p=0.56, p=0.52$, and $\mathrm{p}=0.82$, respectively).

\section{Within-group comparison}

The results showed a statistical decrease in VAS after treatment in group A and B compared to the pre-treatment scores $(\mathrm{p}=0.0001)$. The percent decrease in VAS of group A and B were $80.25 \%$ and $41.93 \%$, respectively.

There was a statistical increase in internal and external rotation ROM after treatment in group A and B compared to pre-treatment ( $\mathrm{p}=0.0001)$. The percent increase in internal and external rotation ROM of group A and B were $22.18 \%, 17.32 \%, 23.45 \%$, and $16.24 \%$, respectively.

\section{Comparison between groups}

The results showed no statistical difference between 
Table 4. Comparison of outcome measures between groups A and B

\begin{tabular}{lcccc}
\hline & Group A & Group B & $\chi^{2}$ & p-value \\
\hline Excellent & $13(65)$ & $3(15)$ & 10.58 & $0.01^{*}$ \\
Good & $2(10)$ & $4(20)$ & & \\
Fair & $2(10)$ & $4(20)$ & & \\
Poor & $3(15)$ & $9(45)$ & & \\
\hline
\end{tabular}

Values are presented as number (\%).

${ }^{*} \mathrm{p}<0.05$.

the groups in VAS, internal rotation, or external rotation ROM before treatment ( $p>0.05)$. There was a statistical decrease in the VAS of group A compared to group B $(p=0.0001)$, while there was no significant difference in internal and external rotation ROM between the groups after treatment ( $p>0.05)$ (Table 3$)$. There was an improvement in outcome measures of group A compared to group B after treatment $(\mathrm{p}=0.01)$ (Table 4$)$.

\section{DISCUSSION}

Sports hernias are more common in athletes who are engaged in sports requiring highly repetitive booting and twisting movements [27]. The exact progression of sport hernia development is still unknown but a combination of several factors may play roles in its development, including balance disturbances between the hip adductors and abdominal muscle power, impairment of the hip ROM, and lack of hip adductors flexibility with repetitive shear force on their attachments at the pelvis [4].

A systematic review of the effectiveness of exercise therapy for groin pain management in athletes concluded that exercise therapy was a key factor in rehabilitation [15]. However, in another review by Swan and Wolcott [28] concerning the diagnosis and operative findings of sports hernias, the authors concluded that surgical interventions provided results that were superior to conventional therapy. Other studies have recommended conventional therapy, including physical rehabilitation with manual therapy for 6 to 8 weeks as the first choice of treatment [29], followed by operative repairs if physical rehabilitation failed and the athlete did not return to pre-injury levels $[30,31]$.

Our study included 40 soccer players with sports hernias who were divided into two equal groups. Both groups received conventional treatment (heat, massage, TENS, and mobilization), while the study group received an ac- tive rehabilitation program. The treatment period lasted 2 months. After the end of treatment, VAS decreased in groups A and B to $80.25 \%$ and $41.93 \%$, respectively. The significant difference in VAS between the groups $(p=0.0001)$ can be attributed to the effect of the active rehabilitation program which aimed to increase strength, pelvic stability, and coordination, and correct the biomechanical abnormalities developed from practicing soccer, which may lead to stress and repetitive trauma to the anatomical structures of the pelvis, subsequently causing pain.

Hip internal and external rotation ROM increased in both groups but there was no statistical difference between the groups after treatment. The improvement in ROM in both groups may be due to decreased pain, stretching exercises, and mobilization techniques which were applied to both groups. However, the post-treatment outcome measures were improved in group A compared to group $B(p=0.01)$. Thirteen patients in group A and only three patients in group B returned to sports activities without groin pain. We believe that core strengthening and balancing exercises played a significant role in the faster return of sportsmen to full activity, hence we suggest that improvement in the target group was due to the type of exercises used.

Several case reports/series have described a proposed rehabilitation protocol for inguinal disruption. Woodward et al. [30] described a three-phase rehabilitation protocol for sports hernia management in a hockey player with groin pain, which included electrotherapy, hydrotherapy, pain modalities, balancing, and strengthening rehabilitative exercises for the trunk, abdominal and hip muscles. By the end of the study, the patient experienced no pain at rest or during activities and was able to return to a full level of activity in National Hockey League competitions for 7 years.

Yuill et al. [17] reported that non-surgical management 
of chronic groin pain, including electrotherapy, pain modalities, and physical rehabilitation exercises for eight weeks increased the muscle power and balance of both the hip and abdomen. By the end of the study, all patients returned to their activities without the recurrence of symptoms at a 2-year follow-up.

Holmich et al. [26] concluded that a rehabilitation program that utilized active exercises could provide an injured athlete with over 10 times the potential to return to active sports. They found that the outcomes of a rehabilitation program that involved active exercises were much better than a passive program. The study found that $79 \%$ of the athletes treated with an active program were able to return to pre-injury status after a seven-month followup, compared to $14 \%$ of athletes treated with a passive program.

There were similarities between these former studies and this study. In the first two studies, the treatment used was a combination of passive and active training programs. The passive program consisted of manual therapy and electrotherapy, while the active training program was directed toward improving strength, core control, coordination, and correcting pelvic muscle imbalance. The latter study compared a passive program with an active training program to treat long-standing groin pain. The results agreed with our results, that active training program was very effective in treating groin pain.

In another case study, rehabilitation of the lower abdominal groin pain was done by structured core muscle retraining. The sportsman was a golfer and the exercise program utilized a stepwise progression in order to increase stability and neuromuscular control. The patient was able to return to the complete practice of golf after four weeks of treatment [27]. The athlete in that study returned to sports in much less time than those in our study. The similarity here was the use of exercises that improved core strength and pelvic stability, indicating their importance in the management of sports hernia.

Sports hernias are usually part of a much wider and serious inguinal disruption injury. Moreover, sports hernias can be caused by many diverse causes which may affect the selection of effective conservative treatment and is considered a limitation of our study. Our study was also limited by small sample size, the absence of muscle strength assessments of the abdominal and adductor muscles, which may have enabled better statistical analy- sis, and the larger amount of treatment time allocated to the treatment group than the control group. In future studies, a follow-up study is necessary to reveal longterm results and recurrence rates.

In conclusion, the results of this study showed that active exercises were a very essential part of sports hernia rehabilitation. An active rehabilitation program was effective for sports hernia management, resulting in decreased pain and a return to sports.

\section{CONFLICT OF INTEREST}

No potential conflict of interest relevant to this article was reported.

\section{AUTHOR CONTRIBUTION}

Conceptualization: Abouelnaga WA. Methodology: Abouelnaga WA, Aboelnour NH. Formal analysis: Aboelnour NH. Funding acquisition: None. Project administration: Abouelnaga WA. Visualization: Aboelnour NH. Writing - original draft: Abouelnaga WA, Aboelnour NH. Writing - review and editing: Aboelnour $\mathrm{NH}$, Abouelnaga WA. Approval of final manuscript: all authors.

\section{REFERENCES}

1. Garvey JF, Hazard H. Sports hernia or groin disruption injury? Chronic athletic groin pain: a retrospective study of 100 patients with long-term follow-up. Herni 2014;18:815-23.

2. Gilmore J. Groin pain in the soccer athlete: fact, fiction, and treatment. Clin Sports Med 1998;17:787-93.

3. Meyers WC, Foley DP, Garrett WE, Lohnes JH, Mandlebaum BR. Management of severe lower abdominal or inguinal pain in high-performance athletes. Am J Sports Med 2000;28:2-8.

4. Hopkins JN, Brown W, Lee CA. Sports hernia: definition, evaluation, and treatment. JBJS Rev 2017;5:e6.

5. St-Onge E, MacIntyre IG, Galea AM. Multidisciplinary approach to non-surgical management of inguinal disruption in a professional hockey player treated with platelet-rich plasma, manual therapy and exercise: a case report. J Can Chiropr Assoc 2015;59:390-7.

6. Draovitch P, Edelstein J, Kelly BT. The layer concept: utilization in determining the pain generators, pathol- 
ogy and how structure determines treatment. Curr Rev Musculoskelet Med 2012;5:1-8.

7. Poultsides LA, Bedi A, Kelly BT. An algorithmic approach to mechanical hip pain. HSS J 2012;8:213-24.

8. Caudill P, Nyland J, Smith C, Yerasimides J, Lach J. Sports hernias: a systematic literature review. $\mathrm{Br} \mathrm{J}$ Sports Med 2008;42:954-64.

9. Cohen B, Kleinhenz D, Schiller J, Tabaddor R. Understanding athletic pubalgia: a review. R I Med J (2013) 2016;99:31-5.

10. Rambani R, Hackney R. Loss of range of motion of the hip joint: a hypothesis for etiology of sports hernia. Muscles Ligaments Tendons J 2015;5:29-32.

11. Preskitt JT. Sports hernia: the experience of Baylor University Medical Center at Dallas. Proc (Bayl Univ Med Cent) 2011;24:89-91.

12. Elattar O, Choi HR, Dills VD, Busconi B. Groin injuries (athletic pubalgia) and return to play. Sports Health 2016;8:313-23.

13. Paksoy M, Sekmen U. Sportsman hernia; the review of current diagnosis and treatment modalities. Ulus Cerrahi Derg 2015;32:122-9.

14. Almeida MO, Silva BN, Andriolo RB, Atallah AN, Peccin MS. Conservative interventions for treating exercise-related musculotendinous, ligamentous and osseous groin pain. Cochrane Database Syst Rev 2013;(6):CD009565.

15. Machotka Z, Kumar S, Perraton LG. A systematic review of the literature on the effectiveness of exercise therapy for groin pain in athletes. Sports Med Arthrosc Rehabil Ther Technol 2009;1:5.

16. Nevin F, Delahunt E. Adductor squeeze test values and hip joint range of motion in Gaelic football athletes with longstanding groin pain. J Sci Med Sport 2014;17:155-9.

17. Yuill EA, Pajaczkowski JA, Howitt SD. Conservative care of sports hernias within soccer players: a case series. J Bodyw Mov Ther 2012;16:540-8.

18. Verrall GM, Slavotinek JP, Fon GT, Barnes PG. Outcome of conservative management of athletic chronic groin injury diagnosed as pubic bone stress injury. Am J Sports Med 2007;35:467-74.

19. Reiman MP, Matheson JW. Restricted hip mobility: clinical suggestions for self-mobilization and muscle re-education. Int J Sports Phys Ther 2013;8:729-40.

20. Page P. Current concepts in muscle stretching for exercise and rehabilitation. Int J Sports Phys Ther 2012;7:109-19.

21. Ross JR, Stone RM, Larson CM. Core muscle injury/ sports hernia/athletic pubalgia, and femoroacetabular impingement. Sports Med Arthrosc Rev 2015;23:21320.

22. Yu SH, Park SD. The effects of core stability strength exercise on muscle activity and trunk impairment scale in stroke patients. J Exerc Rehabil 2013;9:362-7.

23. Kang KY. Effects of core muscle stability training on the weight distribution and stability of the elderly. J Phys Ther Sci 2015;27:3163-5.

24. Amiri B, Sahebozamani M, Sedighi B. The effects of 10-week core stability training on balance in women with multiple sclerosis according to Expanded Disability Status Scale: a single-blinded randomized controlled trial. Eur J Phys Rehabil Med 2019;55:199-208.

25. Dimitrakopoulou A, Schilders E. Sportsman's hernia? An ambiguous term. J Hip Preserv Surg 2016;3:16-22.

26. Holmich P, Uhrskou P, Ulnits L, Kanstrup IL, Nielsen MB, Bjerg AM, et al. Effectiveness of active physical training as treatment for long-standing adductor-related groin pain in athletes: randomised trial. Lancet 1999;353:439-43.

27. Becker LC, Kohlrieser DA. Conservative management of sports hernia in a professional golfer: a case report. Int J Sports Phys Ther 2014;9:851-60.

28. Swan KG Jr, Wolcott M. The athletic hernia: a systematic review. Clin Orthop Relat Res 2007;455:78-87.

29. Kachingwe AF, Grech S. Proposed algorithm for the management of athletes with athletic pubalgia (sports hernia): a case series. J Orthop Sports Phys Ther 2008;38:768-81.

30. Woodward JS, Parker A, Macdonald RM. Non-surgical treatment of a professional hockey player with the signs and symptoms of sports hernia: a case report. Int J Sports Phys Ther 2012;7:85-100.

31. Ekstrand J, Ringborg S. Surgery versus conservative treatment in soccer players with chronic groin pain: a prospective randomised study in soccer players. Eur J Sports Traumatol Rel Res 2001;23:141-5. 